\title{
Dilepton decays of baryon resonances
}

\author{
M. Zétényi* and Gy. Wolf ${ }^{\dagger}$ \\ KFKI Research Institute for Nuclear and Particle Physics, \\ H-1525 Budapest 114, POB. 49, Hungary
}

November 2, 2018

\begin{abstract}
Dalitz decay of baryon resonances is studied and expressions for the decay width are derived for resonances with arbitrary spin and parity. Contributions of the various terms in the transition matrix element are compared and relevance of spin-parity and the resonance mass is discussed. Explicite algebraic expressions are cited for spin $\leq 5 / 2$ resonances. The results can be used in models of dielectron production in elementary reactions and heavy ion collisions.
\end{abstract}

keywords: dileptons, baryon resonances, electromagnetic transitions PACS: 13.30.Ce; 13.40.Hq; 14.20.Gk

\section{Introduction}

The aim of relativistic heavy ion collision experiments is to study the properties of the hot and dense nuclear matter created in the collisions. These properties have to be reconstructed using the information encoded in the particles detected in the final stage of the collision. The best probes for this investigation are dileptons (in the few $\mathrm{GeV}$ per nucleon bombarding energy range dielectrons), which leave the hot and dense zone unaffected by final state interactions.

\footnotetext{
${ }^{*}$ E-mail address: zetenyi@rmki.kfki.hu

†E-mail address: zetenyi@rmki.kfki.hu
} 
Theoretical studies predict a modification of hadron properties (masses and decay widths) in hot and dense nuclear matter. These medium effects can most easily be studied in the case of vector mesons, which decay directly to dileptons and their masses are measured through the invariant mass of the resulting dilepton.

Dielectron production in heavy ion collisions with a few $\mathrm{GeV}$ per nucleon energy has been studied experimentally by the DLS collaboration at BEVALAC and will be studied by the HADES at GSI. In order to obtain a reliable model of heavy ion collisions first one has to check the elementary reaction channels used in the model. For this purpose dilepton production in nucleon-nucleon collisions has to be investigated both experimentally and theoretically.

Particle production in nucleon-nucleon collisions is usually described using the resonance model, i.e., the final state particles are produced via the creation and subsequent decay of baryon resonances. This multistep process is factorized so that initial and final state particles of the individual steps are handled as on-shell particles and in case of resonances their masses are generated according to a Breit-Wigner distribution. Different factorizations result in different models with different elementary channels.

In models of dielectron production in $p p$ collisions usually the following elementary channels are included: direct dileptonic decay of $\rho^{0}, \omega$ and $\phi$ vector mesons, Dalitz decay of mesons $\pi^{0} \rightarrow \gamma e^{+} e^{-}, \eta \rightarrow \gamma e^{+} e^{-}, \omega \rightarrow \pi^{0} e^{+} e^{-}$ and Dalitz decay of the $\Delta(1232)$ resonance, $\Delta \rightarrow N e^{+} e^{-}$. Such models (e.g. [1, 2, 3]) can reasonably well reproduce the experimental dilepton invariant mass spectra in the $E_{b}=1-2 \mathrm{GeV}$ bombarding energy range 4 . In models of heavy ion collisions the additional channels of pion annihilation and $p n$ bremsstrahlung appear.

With increasing beam energy creation of higher mass baryon resonances becomes more and more relevant. In heavy ion collisions kinetic energy of colliding particles can be accumulated in multiple scattering processes, therefore higher mass resonances appear at lower beam energies. These resonances may contribute to the dilepton spectrum through their Dalitz decay similarly to the $\Delta(1232)$. This contribution may especially be relevant in the higher dilepton mass range where the $\rho^{0}$ and $\Delta(1232)$ channels are already negligible. This dilepton mass range coincides with the range of the $\phi$ meson mass.

Recently, production of $\phi$ mesons in subthreshold heavy ion collisions has received some attention both experimentally [5] and theoretically [6, 7]. $\phi$ 
mesons are being studied by the FOPI group at GSI via their kaon decay channel, $\phi \rightarrow K^{+} K^{-}$, but the dileptonic decay channel will also be accessible by the HADES detector. In the latter case an important source of background may come from the Dalitz decay of higher mass resonances.

In this paper we derive expressions for the differential Dalitz decay width of baryon resonances with arbitrary spin-parities. We discuss in detail the Dalitz decay of resonances with spin $\leq 5 / 2$. Our paper is organized as follows: in Sec. 2 we derive expressions for the electromagnetic transition matrix elements of baryon resonances with arbitrary spin-parity and give expressions for the Dalitz decay width. In Sec. 3 we give a detailed discussion of the numerical results. We compare contributions of the various terms in the transition matrix element and discuss the relevance of spin-parity and the resonance mass. In the Appendix we cite the spin projectors used in the calculations and the explicite expressions for the polarization averaged squared transition matrix elements of the baryon resonance Dalitz decays.

\section{Dalitz-decay of a spin- $J$ resonance}

\subsection{Matrix elements of the electromagnetic current}

To study the electromagnetic decays of a spin- $J$ baryon resonance one has to determine the matrix element of the electromagnetic current operator $J_{\mu}$ between the nucleon $(N)$ and the spin- $J$ baryon $(R)$ state $\left\langle N\left|J_{\mu}\right| R\right\rangle$. Let $p_{*}$, $m_{*}$, and $\lambda_{*}$ denote the four-momentum, mass, and helicity of the $R$ resonance, respectively, and $p, m$, and $\lambda$ the corresponding quantities for the nucleon. Let us introduce the notations $q=p_{*}-p$ and $P=\left(p_{*}+p\right) / 2$.

For $J \geq 3 / 2$ the spin- $J$ fermion can be described by a generalized RaritaSchwinger spinor-tensor field $\Psi^{\rho_{1} \cdots \rho_{n}}(n=J-1 / 2)$, therefore the momentumspace wavefunction of the particle is the spinor-tensor amplitude $u^{\rho_{1} \cdots \rho_{n}}\left(p_{*}, \lambda_{*}\right)$. This quantity in general contains some additional lower spin components. The spin- $J$ content of $u^{\rho_{1} \cdots \rho_{n}}\left(p_{*}, \lambda_{*}\right)$ can be selected by prescribing the generalized Rarita-Schwinger relations:

$$
\begin{aligned}
& u^{\cdots \rho_{i} \cdots \rho_{k} \cdots}\left(p_{*}, \lambda_{*}\right)=u^{\cdots \rho_{k} \cdots \rho_{i} \cdots}\left(p_{*}, \lambda_{*}\right), \\
& u^{\cdots \sigma \cdots}{ }_{\sigma} \cdots\left(p_{*}, \lambda_{*}\right)=0, \\
& u^{\cdots \cdots}\left(p_{*}, \lambda_{*}\right) p_{* \sigma}=0, \\
& u^{\cdots \sigma \cdots}\left(p_{*}, \lambda_{*}\right) \gamma_{\sigma}=0 .
\end{aligned}
$$


The matrix element of the electromagnetic current can be written generally as

$$
\left\langle N\left|J_{\mu}\right| R\right\rangle=\bar{u}(p, \lambda) \Gamma_{\mu \rho_{1} \cdots \rho_{n}} u^{\rho_{1} \cdots \rho_{n}}\left(p_{*}, \lambda_{*}\right),
$$

where the form of $\Gamma_{\mu \rho_{1} \cdots \rho_{n}}$ is restricted by the conservation of electric charge, $q^{\mu}\left\langle N\left|J_{\mu}\right| R\right\rangle=0$. The relations (11) and the Dirac equations $\bar{u}(p, \lambda)(\not p-m)=0$ and $\left(\not p_{*}-m_{*}\right) u^{\rho_{1} \cdots \rho_{n}}\left(p_{*}, \lambda_{*}\right)=0$ reduce further the number of independent terms in $\Gamma_{\mu \rho_{1} \cdots \rho_{n}}$ to three, which can be chosen of the form

$$
\Gamma_{\mu \rho_{1} \cdots \rho_{n}}=\sum_{i=1}^{3} f_{i}\left(q^{2}\right) \chi_{\mu \rho_{1}}^{i} p_{\rho_{2}} \cdots p_{\rho_{n}} G,
$$

with

$$
\begin{aligned}
\chi_{\mu \rho}^{1} & =\gamma_{\mu} q_{\rho}-\not q g_{\mu \rho}, \\
\chi_{\mu \rho}^{2} & =P_{\mu} q_{\rho}-(P \cdot q) g_{\mu \rho}, \\
\chi_{\mu \rho}^{3} & =q_{\mu} q_{\rho}-q^{2} g_{\mu \rho},
\end{aligned}
$$

and $G=1$ or $\gamma_{5}$ for resonances with positive or negative normalities, respectively. The normality of a spin- $J$ baryon is defined by $P(-1)^{J-1 / 2}$ with $P$ the intrinsic parity. $f_{i}\left(q^{2}\right), i=1,2,3$ are three independent form factors.

In the spin- $1 / 2$ case the general form of the matrix element of the electromagnetic current is

$$
\left\langle N\left|J_{\mu}\right| R\right\rangle=\bar{u}(p, \lambda) \Gamma_{\mu} u\left(p_{*}, \lambda_{*}\right),
$$

and there are two independent terms in $\Gamma_{\mu}$ :

$$
\Gamma_{\mu}=\sum_{i=1}^{2} f_{i}\left(q^{2}\right) \chi_{\mu}^{i} G
$$

with

$$
\begin{aligned}
& \chi_{\mu}^{1}=(P \cdot q) \gamma_{\mu}-\not P_{\mu}, \\
& \chi_{\mu}^{2}=q^{2} \gamma_{\mu}-\not q q_{\mu} .
\end{aligned}
$$

$G$ is again 1 or $\gamma_{5}$ depending on the normality of the resonance. 


\subsection{The Dalitz-decay width}

The differential width of the Dalitz-decay of a particle is related to its photonic decay width to a virtual photon, $\Gamma_{R \rightarrow N \gamma}(M)$, by 8

$$
\frac{d \Gamma_{R \rightarrow N e^{+} e^{-}}}{d M^{2}}=\frac{\alpha}{3 \pi} \frac{1}{M^{2}} \Gamma_{R \rightarrow N \gamma}(M) .
$$

Here the notation $M^{2}\left(=q^{2}\right)$ is used for the square of the dilepton invariant mass (= mass of the virtual photon). $\Gamma_{R \rightarrow N \gamma}(M)$ can be expressed in terms of the photonic decay matrix element $\langle N \gamma|T| R\rangle$ as

$$
\Gamma_{R \rightarrow N \gamma}(M)=\frac{\sqrt{\lambda\left(m_{*}^{2}, m^{2}, M^{2}\right)}}{16 \pi m_{*}^{3}} \frac{1}{n_{p o l, R}} \sum_{p o l}|\langle N \gamma|T| R\rangle|^{2},
$$

where the transition matrix element is related to the electromagnetic current matrix element by

$$
\langle N \gamma|T| R\rangle=-\epsilon^{\mu}\left\langle N\left|J_{\mu}\right| R\right\rangle,
$$

with $\epsilon^{\mu}$ the photon polarization vector. In (9) $n_{p o l, R}$ is the number of polarization states of the $R$ resonance and $\lambda(a, b, c)=a^{2}+b^{2}+c^{2}-2(a b+b c+a c)$ is the usual kinematical factor.

The polarization sum in (9) runs over all physical polarization states of the incoming and outgoing particles, that is, in the case of the spin- $J$ resonance only over those states that belong to the spin- $J$ content of $u^{\rho_{1} \cdots \rho_{n}}\left(p_{*}, \lambda_{*}\right)$. As a result, the spin- $J$ projector appears in (9) after substituting (2). The projectors for spin-3/2 and 5/2 are given in Appendix A.

If the differential cross section of the production of the resonance $\mathrm{R}$ in an elementary reaction, $d \sigma_{R} / d m_{*}$, is known Eq. (8) can be used to obtain the cross section of dilepton production through the Dalitz decay of $R$ in the same reaction. This differential cross section is given by

$$
\frac{d \sigma_{e^{+} e^{-}, R_{\text {Dalitz }}}}{d M}=\int d m_{*} \frac{d \sigma_{R}}{d m_{*}} \frac{1}{\Gamma_{t o t}^{R}\left(m_{*}\right)} \frac{d \Gamma_{R \rightarrow N e^{+} e^{-}}\left(m_{*}\right)}{d M} .
$$

\subsection{Form factors and coupling constants}

A possibility to determine the functional form of the form factors $f_{i}\left(M^{2}\right)$ is the application of the vector dominance model (VDM) [9]. However, we aim at a model that handles the theoretically more interesting vector meson 
direct dilepton decays separately from the baryon resonance Dalitz decays serving as a background. Applying the VDM to the baryon resonance Dalitz decay channels simultaneously with the inclusion of the vector meson direct decays would give rise to a double counting if - following the usual treatment - the model incorporates vector mesons originating from decays of baryon resonances. The authors of 10 use a sophisticated method to avoid this double counting. In the present paper we do not apply the VDM and keep the form factors $f_{i}\left(q^{2}\right)$ constant.

We introduce the dimensionless coupling constants $g_{i}$ via the equation $f_{i}=e g_{i} / m^{k_{i}}$, where $e$ is the elementary charge related to the fine structure constant by $e^{2}=4 \pi \alpha$. The exponent $k_{i}$ of the nucleon mass is determined by the requirement that the couplings $g_{i}$ are dimensionless. The values of $g_{i}$ are obtained using the real photonic decay width of the resonances.

\section{Discussion of the results}

We first compare the contributions of the various terms in the matrix elements (2) and (5) to the dilepton invariant mass spectrum. For this purpose we have set the dimensionless couplings $g_{i}$ to unity and calculated the contributions of the individual terms and the interference terms to the differential Dalitz decay width. We carried out these calculations for spin $\leq 5 / 2$ resonances and chose a resonance mass of $1.5 \mathrm{GeV}$. The resulting plots are shown in Fig. [1]

We notice that the contribution of terms containing $g_{2}$ in the spin- $1 / 2$ case and terms containing $g_{3}$ in the spin $\geq 3 / 2$ case is free from singularities at $M^{2}=0$. The real photonic width of the resonance $\Gamma_{R \rightarrow N \gamma}(M=0)$ obtained from these terms is zero in accordance with Eq. (8), consequently, the

real photonic decay width cannot be used to fix the coupling constants of these terms. The Dalitz decay width calculated from these terms is significantly smaller than the other contributions throughout the spectrum (with the exception of the $1 / 2-$ case at large dilepton masses), therefore they would need a much larger coupling constant to give a comparable contribution. There seems to be no natural explanation for such a huge difference of the coupling constants. In the following we discard the terms discussed above and make the assumption that in the spin- $1 / 2$ case only the $g_{1}$ term and in the spin $\geq 3 / 2$ case only the $g_{1}$ and $g_{2}$ terms contribute to the Dalitz decay width. 

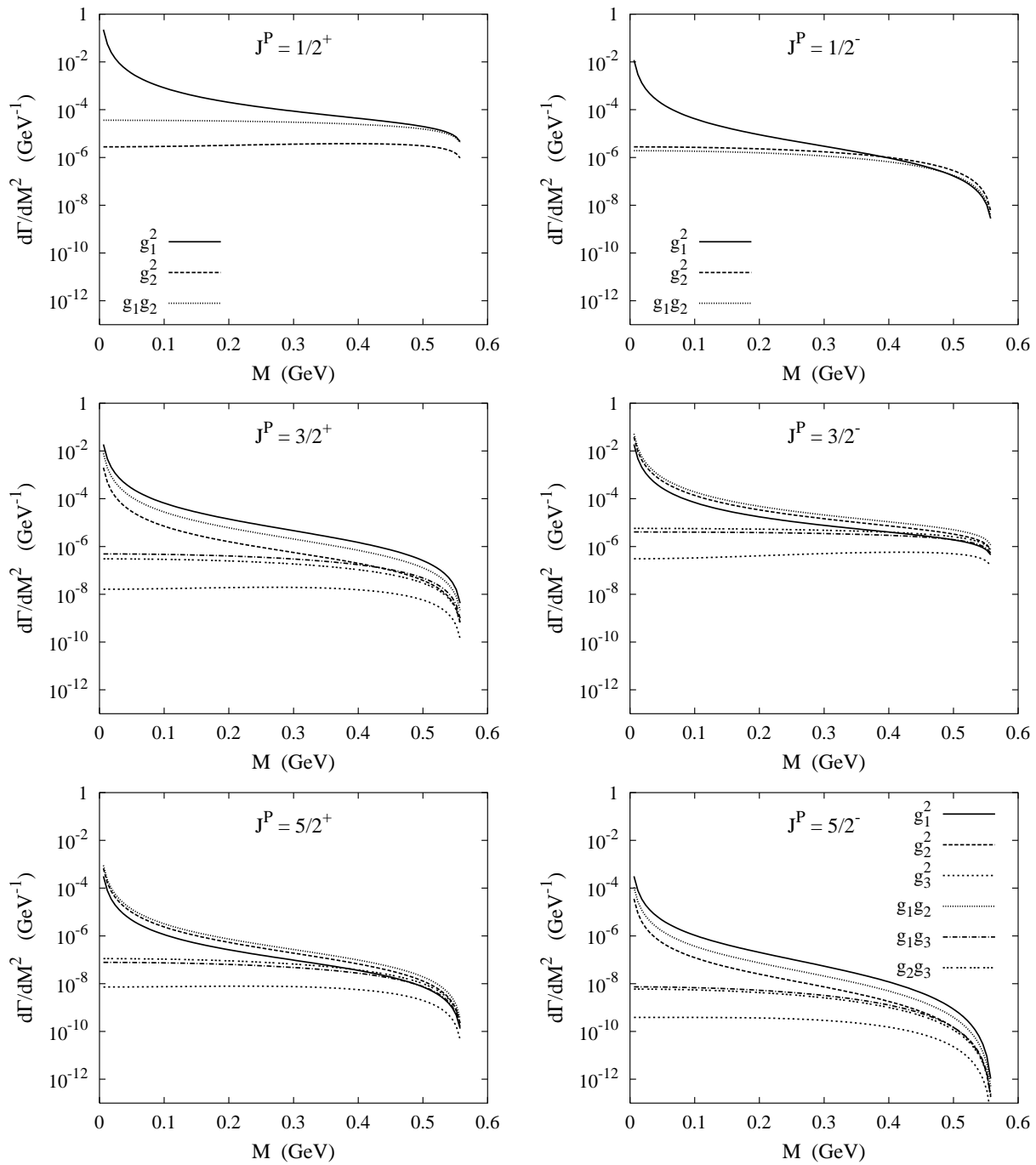

Figure 1: Contributions of the various terms to the differential width of the Dalitz decay $R \rightarrow N e^{+} e^{-}$of an $R$ resonance with mass $m_{*}=1.5 \mathrm{GeV}$ and with various spin-parities. The dimensionless coupling constants are set to unity. The explanation of the linestyles in the Figure for $J^{P}=5 / 2-$ is valid for all plots for $J \geq 3 / 2$. 

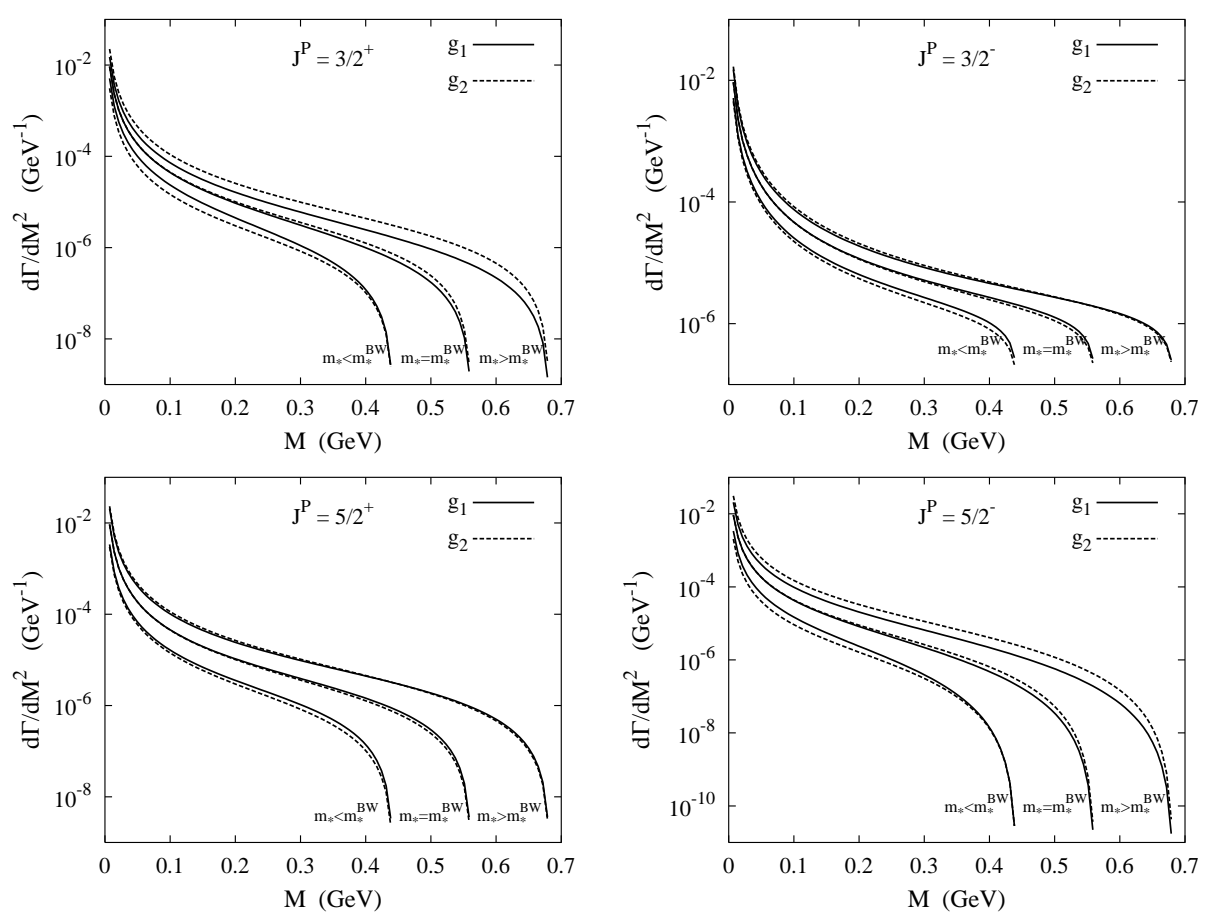

Figure 2: Differential Dalitz decay width of a hypothetical baryon resonance with Breit-Wigner mass $m_{*}^{B W}=1.5 \mathrm{GeV}$, full width $\Gamma_{\text {tot }}=0.12 \mathrm{GeV}$ and a photonic branching ratio of $5 \%$. The two linestyles correspond to the assumptions that only the $g_{1}$ or $g_{2}$ terms contribute $(g 2 \approx 0$ or $g 1 \approx 0)$. Predictions for resonances with masses above and below the Breit-Wigner $\operatorname{mass}\left(m_{*}=m_{*}^{B W} \pm \Gamma_{t o t}\right)$ are also shown. 


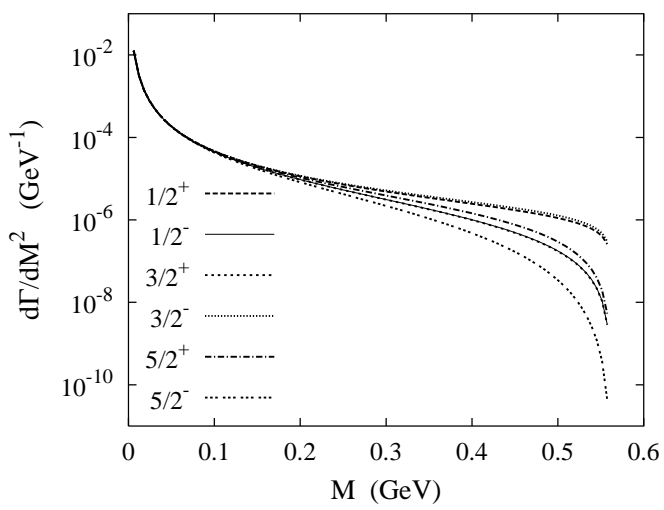

Figure 3: Differential Dalitz decay width of hypothetical baryon resonances with mass $m_{*}=1.5 \mathrm{GeV}$, photonic width $\Gamma_{R \rightarrow N \gamma}=0.006 \mathrm{GeV}$ and with various spin-parities.

In Fig. 2 the differential Dalitz decay width of hypothetical resonances with Breit-Wigner mass $m_{*}^{B W}=1.5 \mathrm{GeV}$, full width $\Gamma_{t o t}=0.12 \mathrm{GeV}$ and a photonic branching ratio of $5 \%$ is shown for various spin-parities ( $\operatorname{spin} \geq 3 / 2)$. The two linestyles refer to the results obtained assuming that only the $g_{1}$ or $g_{2}$ term contributes $\left(g_{2} \approx 0\right.$ or $\left.g_{1} \approx 0\right)$. We also show the results for resonances with considerably larger and smaller mass, specifically, $m_{*}=m_{*}^{B W}+\Gamma_{\text {tot }}$ and $m_{*}=m_{*}^{B W}-\Gamma_{t o t}$. The real photonic width was used to fix the coupling constants in the $m_{*}=m_{*}^{B W}$ case and the same coupling constants have been used in the $m_{*}=m_{*}^{B W} \pm \Gamma_{\text {tot }}$ cases.

For $m_{*}=m_{*}^{B W}$ the $g_{1}$ and $g_{2}$ curves nearly coincide showing that these contributions are practically indistinguishable in the dilepton invariant mass spectrum if the mass of the decaying resonance is close to the Breit-Wigner mass. However, in the spin-parity $3 / 2+$ and $5 / 2-$ cases the difference between the $g_{1}$ and $g_{2}$ contributions is sizable if the resonance mass is well above the Breit-Wigner mass. Since in transport models masses of resonances are usually not far from the Breit-Wigner mass, transition from one of the $g_{1}$ and $g_{2}$ terms to the other or to a linear combination will not have a substantial effect on the predictions for the dilepton invariant mass spectrum. In the following we make the assumption that only the $g_{1}$ term contributes to the Dalitz decay width of baryon resonances. The explicite expressions for the polarization averaged squared transition matrix elements calculated from the 
$g_{1}$ terms are given in Appendix B.

To demonstrate the significance of spin and parity we show in Fig. 3 the differential Dalitz decay width of hypothetical resonances with mass $m_{*}$ $=1.5 \mathrm{GeV}$, photonic decay width $\Gamma_{R \rightarrow N \gamma}=0.006 \mathrm{GeV}$ and with various spin-parities. The invariant mass spectra show large differences in the large dilepton mass range. The largest contribution comes from spin-parity $1 / 2+$ and $3 / 2$ - resonances.

The differential Dalitz decay width of real baryon resonances is shown in Fig. 1 . We included all spin $\leq 5 / 2$ nucleon and $\Delta$ resonances below 2 $\mathrm{GeV}$ listed in the Review of Particle Physics [11] with at least *** status. We assumed that $g_{2}=g_{3}=0$ and fitted the $g_{1}$ coupling constants to the $R^{+} \rightarrow p \gamma$ decay width, the numerical values of which we obtained using the Breit-Wigner full width and the $p \gamma$ branching ratio of the resonances given in [1]]. For the branching ratio, which is poorly known in many cases, we used the average of the lower and upper bounds. In the case of $\Delta$ resonances, where the $p \gamma$ and $n \gamma$ branching ratios are not listed separately, we used the $N \gamma$ partial width to fix the coupling constants. Note that the $p \gamma$ and $n \gamma$ branching ratios may differ significantly.

In Table 11 we give the resulting coupling constants together with the values of the full width and the photonic branching ratio used in the fit. In the case of nucleon resonances we also give the $n \gamma$ couplings.

\section{Conclusion}

In this paper we studied the Dalitz decay of baryon resonances. We have given expressions for the differential Dalitz decay width of resonances with $\operatorname{spin} \leq 5 / 2$. The number of independent terms in the electromagnetic transition matrix element is 2 in the spin- $1 / 2$ case and 3 in the spin $\geq 3 / 2$ case. One of these terms does not contribute to the real photonic decay of the resonances, which is used to fix the coupling constants. We have shown that the remaining two matrix elements in the spin $\geq 3 / 2$ case provide very similar contributions to the dilepton invariant mass spectrum. On the other hand, expressions for different spin-parities give very different dilepton spectra in the large dilepton mass region. Our results can be used in models of dielectron production in elementary hadronic reactions or heavy ion collisions. 

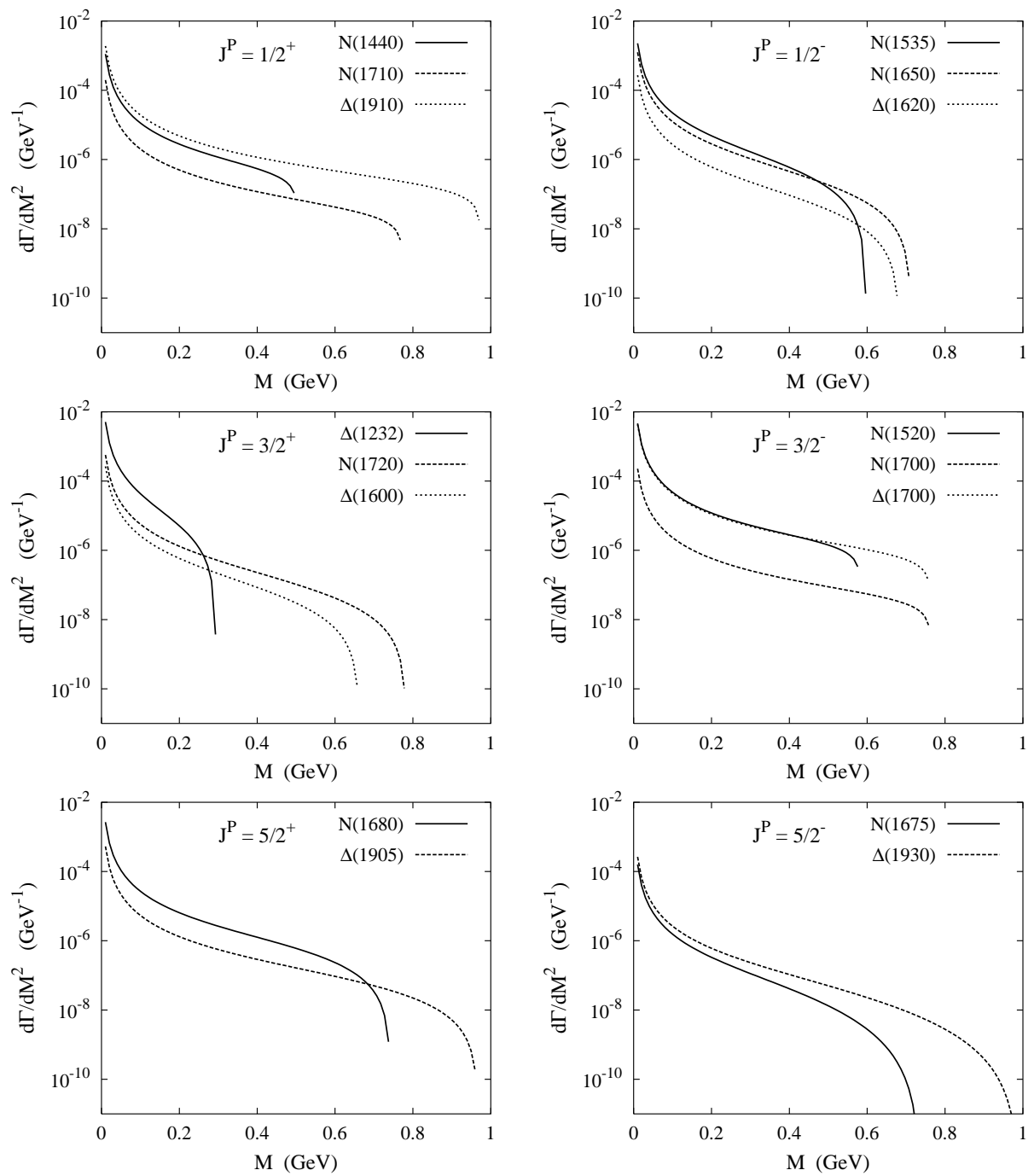

Figure 4: Differential Dalitz decay width of unflavored baryon resonances. The curves correspond to resonances with $m_{*}=m_{*}^{B W}$. The Dalitz decay width of resonances with a mass different from the Breit-Wigner mass can be significantly different (c.f. Fig. 2). 


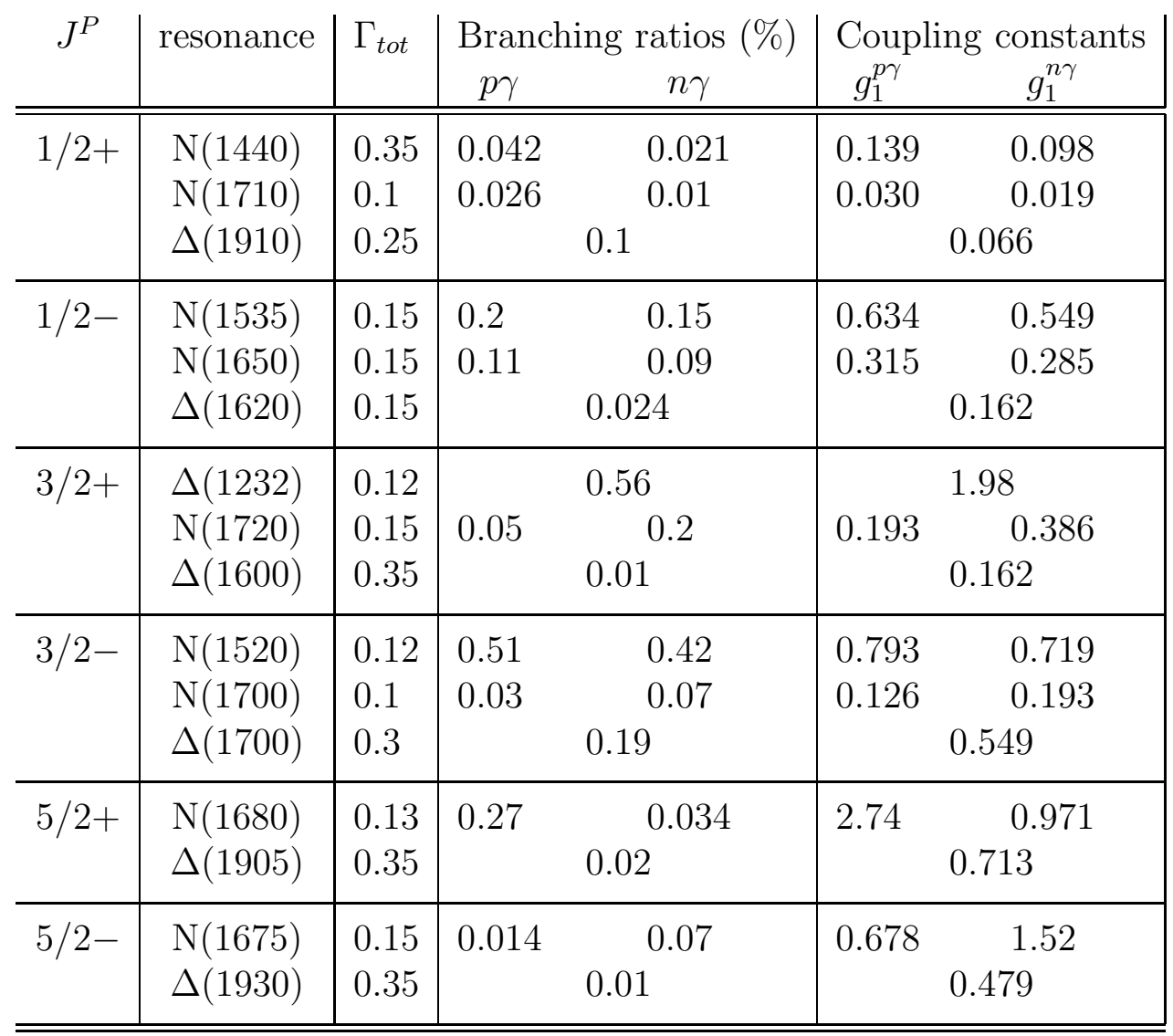

Table 1: Coupling constants $g_{1}$ for the electromagnetic transitions of baryon resonances. In the case of nucleon resonances we give separately the coupling constants for the $p \gamma$ and $n \gamma$ channels. We also give the full widths and photonic branching ratios used to obtain the values of the coupling constants. 


\section{Acknowledgments}

This work was supported by the National Fund for Scientific Research of Hungary, grant Nos. OTKA T30171, T30855 and T32038.

\section{Appendix A}

We give here the spin projectors needed to calculate the square of the transition matrix elements. Throughout the paper we use the conventions of 12 for relativistic quantities and Dirac matrices and spinors. Specifically, we use the normalization $\bar{u}(p, s) u(p, s)=2 m$ for Dirac spinors $\left(p^{2}=m^{2}\right)$, therefore

$$
\sum_{s} u(p, s) \bar{u}(p, s)=\not p+m
$$

The spin-3/2 projector:

$$
\sum_{s} u^{\mu}(p, s) \bar{u}^{\nu}(p, s)=(\not p+m)\left[g^{\mu \nu}-\frac{\gamma^{\mu} \gamma^{\nu}}{3}-\frac{2}{3} \frac{p^{\mu} p^{\nu}}{m^{2}}+\frac{p^{\mu} \gamma^{\nu}-p^{\nu} \gamma^{\mu}}{3 m}\right] .
$$

The spin-5/2 projector:

$$
\begin{aligned}
\sum_{s} & u^{\mu \nu}(p, s) \bar{u}^{\rho \sigma}(p, s)=(\not p+m) \\
\times & {\left[\frac{3}{10}\left(G^{\mu \rho} G^{\nu \sigma}+G^{\mu \sigma} G^{\nu \rho}\right)-\frac{1}{5} G^{\mu \nu} G^{\rho \sigma}\right.} \\
& \left.-\frac{1}{10}\left(T^{\mu \rho} G^{\nu \sigma}+T^{\nu \sigma} G^{\mu \rho}+T^{\mu \sigma} G^{\nu \rho}+T^{\nu \rho} G^{\mu \sigma}\right)\right]
\end{aligned}
$$

with

and

$$
G^{\mu \nu}=-g^{\mu \nu}+\frac{p^{\mu} p^{\nu}}{m^{2}}
$$

$$
T^{\mu \nu}=-\frac{1}{2}\left(\gamma^{\mu} \gamma^{\nu}-\gamma^{\nu} \gamma^{\mu}\right)+\frac{p^{\mu}\left(\not p \gamma^{\nu}-\gamma^{\nu} \not p\right)}{2 m^{2}}-\frac{p^{\nu}\left(\not p \gamma^{\mu}-\gamma^{\mu} \not p\right)}{2 m^{2}}
$$

\section{Appendix B}

In Eq. (9) the squared transition matrix element averaged over initial and summed over final polarization states can be written as

$$
\frac{1}{n_{p o l, R}} \sum_{p o l}|\langle N \gamma|T| R\rangle|^{2}=\left(-g^{\mu \nu}+\frac{q^{\mu} q^{\nu}}{q^{2}}\right) \mathcal{M}_{\mu \nu}
$$


where the notation

$$
\mathcal{M}_{\mu \nu}=\frac{1}{n_{p o l, R}} \sum_{p^{2} l_{N, R}}\left\langle N\left|J_{\mu}\right| R\right\rangle\left\langle R\left|J_{\nu}\right| N\right\rangle
$$

was used. Following [8] we introduce the notations

$$
\begin{aligned}
L_{\mu} & =\left(p_{*} \cdot q\right) q_{\mu}-q^{2} p_{* \mu}, \\
L_{\mu \nu} & =\frac{L_{\mu} L_{\nu}}{L^{2}} \\
T_{\mu \nu} & =g_{\mu \nu}-\frac{q^{\mu} q^{\nu}}{q^{2}}-L_{\mu \nu} .
\end{aligned}
$$

$\mathcal{M}_{\mu \nu}$ can be decomposed in terms of transverse and longitudinal parts, $\mathcal{M}_{T}$ and $\mathcal{M}_{L}$, in the form

$$
\mathcal{M}_{\mu \nu}=-\mathcal{M}_{T} T_{\mu \nu}-\mathcal{M}_{L} L_{\mu \nu}
$$

In terms of $\mathcal{M}_{T}$ and $\mathcal{M}_{L}$ the polarization averaged squared transition matrix element is expressed as

$$
\frac{1}{n_{\text {pol, }, R}} \sum_{\text {pol }}|\langle N \gamma|T| R\rangle|^{2}=2 \mathcal{M}_{T}+\mathcal{M}_{L} .
$$

Here we give the contributions of the $g_{1}^{2}$ terms to $\mathcal{M}_{T}$ and $\mathcal{M}_{L}$ for the various spin-parity cases:

$$
\begin{aligned}
\mathcal{M}_{T}^{1 / 2+}= & 4 \pi \alpha g_{1}^{2} \frac{1}{2 m^{4}}\left(m_{*}^{2}-m^{2}\right)^{2}\left[\left(m_{*}+m\right)^{2}-M^{2}\right] \\
\mathcal{M}_{L}^{1 / 2+}= & 4 \pi \alpha g_{1}^{2} \frac{M^{2}}{2 m^{4}}\left(m_{*}+m\right)^{2}\left[\left(m_{*}+m\right)^{2}-M^{2}\right] \\
\mathcal{M}_{T}^{1 / 2-}= & 4 \pi \alpha g_{1}^{2} \frac{1}{2 m^{4}}\left(m_{*}^{2}-m^{2}\right)^{2}\left[\left(m_{*}-m\right)^{2}-M^{2}\right] \\
\mathcal{M}_{L}^{1 / 2-}= & 4 \pi \alpha g_{1}^{2} \frac{M^{2}}{2 m^{4}}\left(m_{*}-m\right)^{2}\left[\left(m_{*}-m\right)^{2}-M^{2}\right] \\
\mathcal{M}_{T}^{3 / 2+}= & 4 \pi \alpha g_{1}^{2} \frac{1}{12 m_{*}^{2} m^{2}}\left[\left(m_{*}-m\right)^{2}-M^{2}\right] \\
& \times\left(3 m_{*}^{4}+6 m_{*}^{3} m+4 m_{*}^{2} m^{2}+2 m_{*} m^{3}+m^{4}\right. \\
& \left.-2 m_{*} m M^{2}-2 m^{2} M^{2}+M^{4}\right),
\end{aligned}
$$




$$
\begin{aligned}
\mathcal{M}_{L}^{3 / 2+}= & 4 \pi \alpha g_{1}^{2} \frac{M^{2}}{3 m^{2}}\left[\left(m_{*}-m\right)^{2}-M^{2}\right] \\
\mathcal{M}_{T}^{3 / 2-}= & 4 \pi \alpha g_{1}^{2} \frac{1}{12 m_{*}^{2} m^{2}}\left[\left(m_{*}+m\right)^{2}-M^{2}\right] \\
& \times\left(3 m_{*}^{4}-6 m_{*}^{3} m+4 m_{*}^{2} m^{2}-2 m_{*} m^{3}+m^{4}\right. \\
& \left.+2 m_{*} m M^{2}-2 m^{2} M^{2}+M^{4}\right) \\
\mathcal{M}_{L}^{3 / 2-}= & 4 \pi \alpha g_{1}^{2} \frac{M^{2}}{3 m^{2}}\left[\left(m_{*}+m\right)^{2}-M^{2}\right] \\
\mathcal{M}_{T}^{5 / 2+}= & 4 \pi \alpha g_{1}^{2} \frac{1}{480 m_{*}^{4} m^{4}}\left[\left(m_{*}-m\right)^{2}-M^{2}\right]\left[\left(m_{*}+m\right)^{2}-M^{2}\right]^{2} \\
& \times\left(2 m_{*}^{4}-4 m_{*}^{3} m+3 m_{*}^{2} m^{2}-2 m_{*} m^{3}+m^{4}\right. \\
& \left.+2 m_{*} m M^{2}-2 m^{2} M^{2}+M^{4}\right) \\
= & 4 \pi \alpha g_{1}^{2} \frac{M^{2}}{120 m_{*}^{2} m^{4}}\left[\left(m_{*}-m\right)^{2}-M^{2}\right]\left[\left(m_{*}+m\right)^{2}-M^{2}\right]^{2} \\
\mathcal{M}_{L}^{5 / 2+} & 4 \pi \alpha g_{1}^{2} \frac{1}{480 m_{*}^{4} m^{4}}\left[\left(m_{*}-m\right)^{2}-M^{2}\right]^{2}\left[\left(m_{*}+m\right)^{2}-M^{2}\right] \\
\mathcal{M}_{T}^{5 / 2-}= & \times\left(2 m_{*}^{4}+4 m_{*}^{3} m+3 m_{*}^{2} m^{2}+2 m_{*} m^{3}+m^{4}\right. \\
& \left.-2 m_{*} m M^{2}-2 m^{2} M^{2}+M^{4}\right) \\
= & 4 \pi \alpha g_{1}^{2} \frac{M^{2}}{120 m_{*}^{2} m^{4}}\left[\left(m_{*}-m\right)^{2}-M^{2}\right]^{2}\left[\left(m_{*}+m\right)^{2}-M^{2}\right] .(33 \\
\mathcal{M}_{L}^{5 / 2-}= & \\
= &
\end{aligned}
$$

\section{References}

[1] E.L. Bratkovskaya, W. Cassing, M. Effenberger, U. Mosel, Nucl. Phys. A653 (1999) 301.

[2] E.L. Bratkovskaya, W. Cassing, U. Mosel, Nucl. Phys. A686 (2001) 568.

[3] C. Ernst, S.A. Bass, M. Belkacem, H. Stöcker, and W. Greiner, Phys. Rev. C58 (1998) 447.

[4] W.K. Wilson et al., Phys. Rev. C57 (1998) 1865. 
[5] R. Kotte, proceedings of the International workshop XXVIII on Gross Properties of Nuclei and Nuclear Excitations, Hadrons in Dense Matter, Hirschegg, Austria, January 16-22, 2000, eds M. Buballa et al., p. 112.

[6] W.S. Chung, G.Q. Li, and C.M. Ko, Nucl Phys A625 (1997) 347.

[7] H.W. Barz, M. Zétényi, Gy. Wolf, B. Kämpfer, Nucl Phys A, in press.

[8] B.E. Lautrop and J. Smith, Phys Rev. D3 (1971) 1122.

[9] A.T. Titov, B. Kämpfer, E.L. Bratkovskaya, Phys. Rev. C51 (1995) 227.

[10] A. Faessler, C. Fuchs, M.I. Krivoruchenko and B.V. Martemyanov, preprint nucl-th/0010056.

[11] D.E. Groom et al., The European Physical Journal C15 (2000) 1.

[12] J.D. Bjorken and S.D. Drell, Relativistic Quantum Fields, McGraw-Hill Book Company, New York, 1965. 